\title{
Constant Curvature Translation Surfaces in Galilean 3-Space
}

\author{
Muhittin Evren Aydın*, Mihriban Alyamaç Külahçı and Alper Osman \\ Öğrenmiş
}

(Communicated by Kazım İlarslan)

\begin{abstract}
There are five different types of translation surfaces in a Galilean 3-space based upon planarity of generating curves and absolute figure. We obtain these surfaces with arbitrary constant Gaussian and mean curvature, except the type that both of generating curves are non-planar.
\end{abstract}

Keywords: Galilean space; translation surface; Gaussian curvature; mean curvature.

AMS Subject Classification (2010): Primary: 53A35; Secondary: 53B25; 53C42.

\section{Introduction and Preliminaries}

Among the family of surfaces in classic differential geometry translation surfaces have been commonly examined since early 1900s and for that reason an extensive literature relating to these appears. For example see [1, 4, 5, 10, 11], [17], [19]-[24], [30]-[32], [37]-[42]. Such surfaces are geometrically described as translating two curves along each other up to isometries of the ambient space. As far as we know the counterparts of this notion in a Galilean space $\mathbb{G}_{3}$ were firstly considered in Sipus and Divjak's work [27] by providing translation surfaces with constant Gaussian $(K)$ and mean curvature $(H)$ under the condition that the generating curves lie in perpendicular planes. Extending this condition, which is our motivation for the present study, leads us to open fields for further investigations. More precisely, by assuming $K=$ const. and $H=$ const. we shall present the translation surfaces in $\mathbb{G}_{3}$, except the ones whose both of generating curves are space curves.

A Cayley-Klein 3-space is defined as a projective 3-space $P_{3}(\mathbb{R})$ with certain absolute figure. Group of motions of this space are introduced by the projective transformations which leave invariant the absolute figure. Metrically arguments given by the absolute figure are invariant under this group (cf. [28]). The Galilean 3-space $\mathbb{G}_{3}$ is one of real Cayley-Klein 3-spaces with the absolute figure $\{\Gamma, l, \iota\}$, where $\Gamma$ is a plane (absolute plane) in $P_{3}(\mathbb{R}), l$ a line (absolute line) in $\Gamma$ and $\iota$ is the fixed elliptic involution of the points of $l$. For technical details, we refer the reader to [2, 3], [6]-[9], [12]-[15], [18, 25, 26, 29], [33]-[36], [43]. Let $\left(x_{0}: x_{1}: x_{2}: x_{3}\right)$ denote the homogeneous coordinates in $P_{3}(\mathbb{R})$. Then $\Gamma$ is characterized by $x_{0}=0, l x_{0}=x_{1}=0$ and $\iota$

$$
\left(x_{0}: x_{1}: x_{2}: x_{3}\right) \longmapsto\left(x_{0}: x_{1}: x_{3}:-x_{2}\right) .
$$

For an affine model of $\mathbb{G}_{3}$ that is our interest field we use affine coordinates instead of the homogeneous coordinates. Then, by means of the affine coordinates, the group of motions of $\mathbb{G}_{3}$ is given by the transformation

$$
(x, y, z) \longmapsto\left(x^{\prime}, y^{\prime}, z^{\prime}\right):\left\{\begin{array}{l}
x^{\prime}=a+x, \\
y^{\prime}=b+c x+(\cos \theta) y+(\sin \theta) z, \\
z^{\prime}=d+e x-(\sin \theta) y+(\cos \theta) z,
\end{array}\right.
$$


where $a, b, c, d, e$ and $\theta$ are some constants. For given points $p=\left(p_{1}, p_{2}, p_{3}\right)$ and $q=\left(q_{1}, q_{2}, q_{3}\right)$, the Galilean distance is introduced by the absolute figure, namely

$$
d(p, q)= \begin{cases}\left|q_{1}-p_{1}\right|, & \text { if } p_{1} \neq q_{1}, \\ \sqrt{\left(p_{2}-q_{2}\right)^{2}+\left(p_{3}-q_{3}\right)^{2}}, & \text { otherwise. }\end{cases}
$$

Lines and planes in $\mathbb{G}_{3}$ are categorized up to the absolute figure. Explicitly, a line is said to be non-isotropic (resp. isotropic) if its intersection with the absolute line $l$ is empty (resp. non-empty). Contrary to this, a plane is said to be isotropic if it does not involve l, otherwise it is said to be Euclidean. In other words, an isotropic plane does not involve any isotropic direction. In the affine model of $\mathbb{G}_{3}$, the Euclidean planes are determined by the equation $x=$ const. Accordingly, a vector is called isotropic if it is involved in the Euclidean plane $x=0$. Non-isotropic vectors are of the form $u=\left(u_{1} \neq 0, u_{2}, u_{3}\right), u_{1}, u_{2}, u_{3} \in \mathbb{R}$.

For given two vectors $u=\left(u_{1}, u_{2}, u_{3}\right)$ and $v=\left(v_{1}, v_{2}, v_{3}\right)$ in $\mathbb{G}_{3}$, the Galilean angle $\theta$ between them is defined as the Euclidean angle if $u$ and $v$ are isotropic. Otherwise, it is defined as the Galilean distance. Nevertheless, all non-isotropic vectors are orthogonal to the isotropic ones.

A curve given in parametric form $\alpha=\alpha(s)=(x(s), y(s), z(s))$ is said to be non-isotropic (or admissible) if nowhere its tangent vector is isotropic, namely $x^{\prime}(s)=\frac{d x}{d s} \neq 0$. Otherwise the curve $\alpha$ is said to be isotropic. Let $\alpha$ be a non-isotropic curve having unit speed (i.e. $x^{\prime}(s)= \pm 1$ ). In this case the parameter $s$ is called arc-length parameter of $\alpha$. Hence the curvature and the torsion are given by

$$
\kappa(s)=\sqrt{\left[y^{\prime \prime}(s)\right]^{2}+\left[z^{\prime \prime}(s)\right]^{2}}, \tau(s)=\frac{\operatorname{det}\left(\alpha^{\prime}(s), \alpha^{\prime \prime}(s), \alpha^{\prime \prime \prime}(s)\right)}{[\kappa(s)]^{2}}(\kappa(s) \neq 0) .
$$

If $\tau(s)=0$ for all $s$, then it implies $y^{\prime \prime} z^{\prime \prime \prime}-y^{\prime \prime \prime} z^{\prime \prime}=0$. In this sense, we have the following cases:

1. $y^{\prime \prime}=0$ and $z^{\prime \prime} \neq 0$. Then it follows $y(s)=a s+b, a, b \in \mathbb{R}$ i.e. $\alpha$ lies in a plane of the form $y=a x+b$;

2. $z^{\prime \prime}=0$ and $y^{\prime \prime} \neq 0$. Then it follows $z(s)=a s+b, a, b \in \mathbb{R}$ i.e. $\alpha$ lies in a plane of the form $z=a x+b$;

3. $z^{\prime \prime}=a y^{\prime \prime}$ for $a \in \mathbb{R}, a \neq 0$. Then it follows $z(s)=a y(s)+b s+c, b, c \in \mathbb{R}$ i.e. $\alpha$ lies in a plane of the form $z=a y+b x+c$.

We briefly call a curve planar (resp. space curve) provided $\tau(s)=0$ (resp. $\tau(s) \neq 0$ ) for all $s$. Obviously; the space curves are non-isotropic, whereas the isotropic curves are Euclidean planar, that is, lie in the Euclidean plane $x=$ const.

A surface in $\mathbb{G}_{3}$ is parameterized by the mapping

$$
r: D \subseteq \mathbb{R}^{2} \longrightarrow \mathbb{G}_{3},\left(u_{1}, u_{2}\right) \longmapsto\left(x\left(u_{1}, u_{2}\right), y\left(u_{1}, u_{2}\right), z\left(u_{1}, u_{2}\right)\right) .
$$

In order to indicate the partial derivatives we use

$$
x_{, i}=\frac{\partial x}{\partial u_{i}} \text { and } x_{, i j}=\frac{\partial^{2} x}{\partial u_{i} \partial u_{j}}, 1 \leq i, j \leq 2 .
$$

Then $r$ is said to be admissible if nowhere it has Euclidean tangent planes, i.e., $x, i \neq 0$ for some $i=1,2$. The first fundamental form is given by

$$
d s^{2}=\left(g_{1} d u_{1}+g_{2} d u_{2}\right)^{2}+\varepsilon\left(h_{11} d u_{1}^{2}+2 h_{12} d u_{1} d u_{2}+h_{22} d u_{2}^{2}\right),
$$

where $g_{i}=x_{, i}, h_{i j}=y_{, i} y_{, j}+z_{, i} z_{, j}, i, j=1,2$, and

$$
\varepsilon= \begin{cases}0, & \text { if the direction } d u_{1}: d u_{2} \text { is non-isotropic, } \\ 1, & \text { if the direction } d u_{1}: d u_{2} \text { is isotropic. }\end{cases}
$$

Let us introduce a function $W$ given by

$$
W=\sqrt{\left(x_{, 1} z_{, 2}-x_{, 2} z_{, 1}\right)^{2}+\left(x_{, 2} y_{, 1}-x_{, 1} y_{, 2}\right)^{2}} .
$$

Then the normal vector field is defined as

$$
N=\frac{1}{W}\left(0,-x_{, 1} z_{, 2}+x_{, 2} z_{, 1}, x_{, 1} y_{, 2}-x_{, 2} y_{, 1}\right)
$$


and thereafter the second fundamental form

$$
I I=L_{11} d u_{1}^{2}+2 L_{12} d u_{1} d u_{2}+L_{22} d u_{2}^{2},
$$

where

$$
L_{i j}=\frac{1}{g_{1}}\left(g_{1}\left(0, y_{, i j}, z_{, i j}\right)-g_{i, j}\left(0, y_{, 1}, z_{, 1}\right)\right) \cdot N, g_{1} \neq 0
$$

or

$$
L_{i j}=\frac{1}{g_{2}}\left(g_{2}\left(0, y_{, i j}, z_{, i j}\right)-g_{i, j}\left(0, y_{, 2}, z_{, 2}\right)\right) \cdot N, g_{2} \neq 0 .
$$

Note that the dot". " denotes the Euclidean scalar product. Thereby, the Gaussian and the mean curvature are defined as

$$
K=\frac{L_{11} L_{22}-L_{12}^{2}}{W^{2}} \text { and } H=\frac{g_{2}^{2} L_{11}-2 g_{1} g_{2} L_{12}+g_{1}^{2} L_{22}}{2 W^{2}} .
$$

A surface is said to be minimal (resp. flat) if its mean (resp. Gaussian) curvature vanishes. Therefore the minimal surfaces in $\mathbb{G}_{3}$ are classified by the following result (see [35] ):

Theorem 1.1. [35] Minimal surfaces in $\mathbb{G}_{3}$ are cones whose vertices lie on the absolute line and the ruled surfaces of type C. They are all conoidal ruled surfaces having the absolute line as the directional line in infinity.

Remark 1.1. A ruled surface of type $C$ in $\mathbb{G}_{3}$ is parameterized by

$$
r\left(u_{1}, u_{2}\right)=\left(u_{1}, x\left(u_{1}\right)+u_{2} y\left(u_{1}\right), u_{2} z\left(u_{1}\right)\right),
$$

for smooth functions $x, y, z$ of one variable.

\section{Translation Surfaces}

A translation surface in $\mathbb{G}_{3}$ is locally parameterized by

$$
r: I_{1} \times I_{2} \subseteq \mathbb{R}^{2} \longrightarrow \mathbb{G}_{3}, r(u, v)=\alpha(u)+\beta(v),
$$

where $\alpha$ and $\beta$ are so-called generating curves. Under the condition that $\alpha$ and $\beta$ are planar, the authors in [27] categorized such a surface up to the absolute figure:

type $1 \alpha$ is planar non-isotropic curve and $\beta$ isotropic curve,

type $2 \alpha$ and $\beta$ are planar non-isotropic curves.

If the planes involving the generating curves are chosen as perpendicular to each other, the surfaces of type 1 and type 2 are respectively parameterized by

$$
r(u, v)=(u, v, f(u)+g(v))(\text { type } 1), r(u, v)=(u+v, g(v), f(u)) \text { (type 2). }
$$

These surfaces with $K=$ const. and $H=$ const. were obtained in [27]. Otherwise, i.e. the planes are not perpendicular, then the notion of affine translation surface arises, introduced by Liu and Yu [20] as the graph surfaces of the form

$$
z(u, v)=f(u)+g(v+a u), a \in \mathbb{R}, a \neq 0 .
$$

By following this, we call the surfaces of type 1 and type 2 affine translation surfaces. We classify such surfaces in Section 3 with $K=$ const. and $H=$ const. Furthermore, the generating curves could be non-planar and hereinafter it is necessary to extend above categorization:

type $3 \alpha$ is isotropic curve and $\beta$ space curve,

type $4 \alpha$ is planar non-isotropic curve and $\beta$ space curve,

type $5 \alpha$ and $\beta$ are space curves.

We also provide the surfaces of type 3 and type 4 in next sections with $K=$ const. and $H=$ const. 


\section{Constant Curvature Affine Translation Surfaces}

Assume that $A=\left(a_{i j}\right)$ is a real invertible matrix, $i, j=1,2$, and $w=\operatorname{det} A \neq 0$. Let us consider the following planar curves:

$$
\begin{cases}\alpha=\alpha(u)=\left(\frac{a_{22}}{w} u, \frac{-a_{21}}{w} u, f(u)\right), & P_{\alpha}: a_{21} x+a_{22} y=0, \\ \beta=\beta(v)=\left(\frac{-a_{12}}{w} v, \frac{a_{11}}{w} v, g(v)\right), & P_{\beta}: a_{11} x+a_{12} y=0,\end{cases}
$$

where $P_{\alpha}$ and $P_{\beta}$ denote the planes containing the curves $\alpha, \beta$. It is easily seen that $P_{\alpha}$ is perpendicular to $P_{\beta}$ provided $A$ is an orthogonal matrix. Nevertheless, if $a_{12}=0$ (resp. $a_{22}=0$ ) in (3.1) then $\beta$ (resp. $\alpha$ ) becomes an isotropic curve. Otherwise i.e. $a_{12} a_{22} \neq 0$, both of them are non-isotropic curves.

By a translation of $\alpha$ and $\beta$, we derive the following admissible surface:

$$
r(u, v)=\left(\frac{a_{22}}{w} u-\frac{a_{12}}{w} v, \frac{a_{11}}{w} v-\frac{a_{21}}{w} u, f(u)+g(v)\right),
$$

where $a_{12}^{2}+a_{22}^{2} \neq 0$ because $w \neq 0$. Point out that by changing the coordinates $u=a_{11} x+a_{12} y, v=a_{21} x+a_{22} y$ (3.2) turns to the usual form of affine translation surface given by

$$
r(x, y)=\left(x, y, f\left(a_{11} x+a_{12} y\right)+g\left(a_{21} x+a_{22} y\right)\right) .
$$

The positive side of such a notion is to represent the surfaces of both type 1 and type 2 into one format as well as a natural generalization of the surfaces given by (2.1).

Throughout this section, we only discuss the cases relating to $f$ due to the fact that the roles of $f$ and $g$ are symmetric.

After a calculation, we have the Gaussian curvature:

$$
K=\frac{w^{2} f^{\prime \prime} g^{\prime \prime}}{\left[1+\left(a_{12} f^{\prime}+a_{22} g^{\prime}\right)^{2}\right]^{2}}
$$

where $f^{\prime}=\frac{d f}{d u}$ and $g^{\prime}=\frac{d g}{d v}$, etc.

Theorem 3.1. If the surface in $\mathbb{G}_{3}$ given by (3.2) has constant Gaussian curvature $K_{0}$, then it is either a generalized cylinder with isotropic or non-isotropic rulings $\left(K_{0}=0\right)$; or a certain surface parameterized by, up to suitable translations and constants,

$$
r(u, s)=\left(u, \frac{-a_{21}}{w} u+\frac{s}{2} \sqrt{1-\left(\frac{K_{0}}{2 c}\right) s^{2}}+\frac{c}{2 K_{0}} \arcsin \left(\frac{K_{0}}{c} s\right), \frac{c}{2} u^{2}+\frac{K_{0}}{2 c} s^{2}\right),
$$

where $c \in \mathbb{R}-\{0\}$ and $s$ is the arc-length parameter of $\beta$.

Proof. Assume that $K_{0}=0$. Then (3.4) leads $f$ to be a linear function and thus the surface becomes a generalized cylinder (so-called cylindrical surface, see [16], p. 439) with non-isotropic $\left(a_{22} \neq 0\right)$ or isotropic rulings $\left(a_{22}=0\right)$. Otherwise, i.e. $K_{0} \neq 0$, by (3.4) we get $f^{\prime \prime} g^{\prime \prime} \neq 0$. Taking the partial derivative of (3.4) with respect to $u$ gives

$$
4 K_{0}\left[1+\left(a_{12} f^{\prime}+a_{22} g^{\prime}\right)^{2}\right]\left(a_{12} f^{\prime}+a_{22} g^{\prime}\right)\left(a_{12} f^{\prime \prime}\right)=w^{2} f^{\prime \prime \prime} g^{\prime \prime} .
$$

To solve (3.5), we have two cases:

1. $a_{12}=0$. (3.5) follows that $f^{\prime \prime}=c_{1}, c_{1} \in \mathbb{R}-\{0\}$. Then by (3.4) we get

$$
\frac{K_{0}}{a_{11}^{2} a_{22} c_{1}}=\frac{a_{22} g^{\prime \prime}}{\left[1+\left(a_{22} g^{\prime}\right)^{2}\right]^{2}},
$$

where $a_{11} a_{22} \neq 0$ since $w \neq 0$. We treat the method used in [27] in order to solve (3.6). Since $a_{12}=0, \beta$ is an isotropic curve and its reparametrization having unit speed is given by

$$
\beta(s)=(0, p(s), q(s)),\left(p^{\prime}\right)^{2}+\left(q^{\prime}\right)^{2}=1,
$$


where the prime denotes the derivative with respect to the arc-length parameter. In this case (3.4) turns to

$$
K_{0}=f^{\prime \prime} q^{\prime \prime}
$$

After solving (3.8), we obtain

$$
q(s)=\frac{K_{0}}{2 c_{1}} s^{2}+c_{2} s+c_{3}, c_{2}, c_{3} \in \mathbb{R} .
$$

Morever, we have $f(u)=\frac{c_{1}}{2} u^{2}+c_{4} u+c_{5}, c_{4}, c_{5} \in \mathbb{R}$ because $f^{\prime \prime}=c_{1}$. Up to congruency of $\mathbb{G}_{3}$ we may assume $c_{3}=c_{5}=0$ and, up to suitable translations of $u, s$ choose $c_{2}=c_{4}=0$. Considering it into (3.7) leads to

$$
p(s)=\frac{s}{2} \sqrt{1-\left(\frac{K_{0}}{c_{1}} s\right)^{2}}+\frac{c_{1}}{2 K_{0}} \arcsin \left(\frac{K_{0}}{c_{1}} s\right)+c_{6},
$$

where one may assume $c_{6}=0$ up to congruency of $\mathbb{G}_{3}$. Therefore one is congruent to the surface given in the hypothesis of the theorem.

2. $a_{12} \neq 0$. By the symmetry we have $a_{22} \neq 0$ and then (3.5) can be rewritten as

$$
\left[1+\left(a_{12} f^{\prime}+a_{22} g^{\prime}\right)^{2}\right]\left(a_{12} f^{\prime}+a_{22} g^{\prime}\right)\left(g^{\prime \prime}\right)^{-1}=\frac{w^{2} f^{\prime \prime \prime}}{4 K_{0} a_{12} f^{\prime \prime}} .
$$

The partial derivative of (3.9) with respect to $v$ yields

$$
\frac{1+3\left(a_{12} f^{\prime}+a_{22} g^{\prime}\right)^{2}}{a_{12} f^{\prime}+a_{22} g^{\prime}+\left(a_{12} f^{\prime}+a_{22} g^{\prime}\right)^{3}}=\frac{g^{\prime \prime \prime}}{a_{22}\left(g^{\prime \prime}\right)^{2}} .
$$

Again taking the partial derivative of (3.10) with respect to $u$ gives the following:

$$
1+3\left(a_{12} f^{\prime}+a_{22} g^{\prime}\right)^{4}=0,
$$

which is a contradiction and this completes the proof.

For the mean curvature, we have

$$
H=\frac{a_{12}^{2} f^{\prime \prime}+a_{22}^{2} g^{\prime \prime}}{2\left[1+\left(a_{12} f^{\prime}+a_{22} g^{\prime}\right)^{2}\right]^{\frac{3}{2}}} .
$$

Theorem 3.2. Let the surface in $\mathbb{G}_{3}$ given by (3.2) have constant mean curvature $H_{0}$. Then:

(i) If $H_{0}=0$, it is either

(i.1) an isotropic plane, or

(i.2) a generalized cylinder with isotropic rulings, or

(i.3) a non-cylindrical ruled surface of type $C$ whose the base curve is a parabolic circle.

(ii) Otherwise $\left(H_{0} \neq 0\right)$; it is either

(ii.1) a certain surface given by

$$
r(u, v)=\left(\frac{a_{22}}{w} u, \frac{a_{11}}{w} v-\frac{a_{21}}{w} u, f(u)-\frac{1}{2 H_{0}} \sqrt{1-\left(\frac{2 H_{0}}{a_{22}} v\right)^{2}}\right), a_{22} \neq 0,
$$

(ii.2) or a generalized cylinder with non-isotropic rulings given by

$$
r(u, v)=\left(\frac{a_{22}}{w} u-\frac{a_{12}}{w} v, \frac{a_{11}}{w} v-\frac{a_{21}}{w} u, c u-\frac{1}{2 H_{0}} \sqrt{1-\left(\frac{2 H_{0}}{a_{22}} v\right)^{2}}-\frac{c a_{12}}{a_{22}} v\right), a_{22} \neq 0, c \in \mathbb{R} .
$$


Proof. Let $M^{2}$ be the surface given by (3.2). We divide the proof into two cases:

1. $H_{0}=0$. Then (3.11) reduces to

We have three sub-cases:

$$
a_{12}^{2} f^{\prime \prime}+a_{22}^{2} g^{\prime \prime}=0
$$

(a) $f^{\prime \prime}=0=g^{\prime \prime}$ is a solution for (3.12). This leads $M^{2}$ to be an isotropic plane, which implies the statement (i.1) of the theorem.

(b) $a_{12}=0$. Because $w \neq 0$ we get $a_{22} \neq 0$. Thus (3.12) immediately implies $g^{\prime \prime}=0$ and this proves the statement (i.2) of the theorem.

(c) $a_{12} \neq 0$. The symmetry implies $a_{22} \neq 0$. Solving (3.12) gives

$$
f(u)=\frac{c_{1}}{2 a_{12}^{2}} u^{2}+c_{2}+c_{3}, g(v)=-\frac{c_{1}}{2 a_{22}^{2}} v^{2}+c_{4}+c_{5},
$$

where $c_{1}, \ldots, c_{5} \in \mathbb{R}, c_{1} \neq 0$. Up to congruency of $\mathbb{G}_{3}$ we may assume $c_{3}=c_{5}=0$ and, up to suitable translations of $u, v$ choose $c_{2}=c_{4}=0$. Hence, substituting this into (3.3) leads to

$$
r(x, y)=\left(x, 0, \frac{c_{1}}{2}\left[\left(\frac{a_{11}}{a_{12}}\right)^{2}-\left(\frac{a_{21}}{a_{22}}\right)^{2}\right] x^{2}\right)+y\left(0,1,2 x\left[\frac{a_{11}}{a_{12}}-\frac{a_{21}}{a_{22}}\right]\right) .
$$

This means that $M^{2}$ is congruent to a non-cylindrical ruled surface whose the base curve is a parabolic circle and the rulings are isotropic.

2. $H_{0} \neq 0$. We have two sub-cases:

(a) $a_{12}=0$. Then (3.11) reduces to

$$
H_{0}=\frac{a_{22}^{2} g^{\prime \prime}}{2\left[1+\left(a_{22} g^{\prime}\right)^{2}\right]^{\frac{3}{2}}},
$$

where $a_{22} \neq 0$ because $w \neq 0$. As in previous case, up to suitable translations and constants, solving (3.13) gives

$$
g(v)=-\frac{1}{2 H_{0}} \sqrt{1-\left(\frac{2 H_{0}}{a_{22}} v\right)^{2}} .
$$

Therefore we obtain that $M^{2}$ is congruent to the surface given in the statement (ii.1) of the theorem. (b) $a_{12} \neq 0$. Taking partial derivative of (3.11) with respect to $u$ gives

$$
6 H_{0}\left[1+\left(a_{12} f^{\prime}+a_{22} g^{\prime}\right)^{2}\right]^{\frac{1}{2}}\left[a_{12} f^{\prime}+a_{22} g^{\prime}\right]\left[a_{12} f^{\prime \prime}\right]=a_{12}^{2} f^{\prime \prime \prime} .
$$

For (3.14) there are two possibilities:

i. $f^{\prime \prime}=0$. Then from (3.11), we have

$$
\frac{2 H_{0}}{a_{22}}=\frac{a_{22} g^{\prime \prime}}{\left[1+\left(a_{12} c_{6}+a_{22} g^{\prime}\right)^{2}\right]^{\frac{3}{2}}},
$$

where $f^{\prime}=c_{6}, c_{6} \in \mathbb{R}$. By solving (3.15), up to suitable translations and constants, we obtain

$$
g(v)=-\frac{1}{2 H_{0}} \sqrt{1-\left(\frac{2 H_{0}}{a_{22}} v\right)^{2}}-\frac{c_{6} a_{12}}{a_{22}} v .
$$

Therefore $M^{2}$ is congruent to the surface given in the statement (ii.2) of the theorem.

ii. $f^{\prime \prime} \neq 0$. Then (3.14) can be rewritten as

$$
\left[1+\left(a_{12} f^{\prime}+a_{22} g^{\prime}\right)^{2}\right]^{\frac{1}{2}}\left[a_{12} f^{\prime}+a_{22} g^{\prime}\right]=\frac{a_{12} f^{\prime \prime \prime}}{6 H_{0} f^{\prime \prime}} .
$$

The partial derivative of (3.16) with respect to $v$ gives

$$
1+2\left(a_{12} f^{\prime}+a_{22} g^{\prime}\right)^{2}=0,
$$

which is a contradiction. This completes the proof. 


\section{Constant Curvature Surfaces of Type 3}

Let one generating curve be the space curve given by $\alpha=\alpha(u)=\left(u, f_{1}(u), f_{2}(u)\right)$ and another one the unit speed isotropic curve given by

$$
\left\{\begin{array}{l}
\beta=\beta(v)=\left(0, g_{1}(v), g_{2}(v)\right), \\
\left(g_{1}^{\prime}\right)^{2}+\left(g_{2}^{\prime}\right)^{2}=1, g_{i}^{\prime}=\frac{d g_{i}}{d v}, i=1,2 .
\end{array}\right.
$$

Here we may assume $g_{1}^{\prime} \neq 0$ without loss of generality. The last equality implies

$$
g_{1}^{\prime} g_{1}^{\prime \prime}+g_{2}^{\prime} g_{2}^{\prime \prime}=0 .
$$

Because the torsion of $\alpha$ is different from zero we get

$$
f_{1}^{\prime \prime} f_{2}^{\prime \prime \prime}-f_{1}^{\prime \prime \prime} f_{2}^{\prime \prime} \neq 0
$$

where $\frac{d f_{i}}{d u}=f_{i}^{\prime}$, etc. $i=1,2$. Thereby the obtained translation surface belongs to type 3 and given by

$$
r(u, v)=\left(u, f_{1}(u)+g_{1}(v), f_{2}(u)+g_{2}(v)\right) .
$$

By a calculation, the Gaussian curvature is

$$
K=-\frac{g_{2}^{\prime \prime}}{g_{1}^{\prime}}\left(f_{1}^{\prime \prime} g_{2}^{\prime}-f_{2}^{\prime \prime} g_{1}^{\prime}\right)
$$

Theorem 4.1. If the surface in $\mathbb{G}_{3}$ given by (4.3) has constant Gaussian curvature $K_{0}$, then it is a generalized cylinder with isotropic rulings $\left(K_{0}=0\right)$.

Proof. The proof is by contradiction. Then $K_{0} \neq 0$ implies $g_{2}^{\prime \prime} \neq 0$. Hence (4.4) can be rewritten as

$$
-K_{0} \frac{g_{1}^{\prime}}{g_{2}^{\prime \prime}}=f_{1}^{\prime \prime} g_{2}^{\prime}-f_{2}^{\prime \prime} g_{1}^{\prime}
$$

The partial derivative of (4.5) with respect to $u$ follows

$$
f_{1}^{\prime \prime \prime} g_{1}^{\prime}-f_{2}^{\prime \prime \prime} g_{2}^{\prime}=0
$$

$g_{1}^{\prime}$ and $g_{2}^{\prime}$ in (4.6) can not be linearly independent due to (4.2) and thus it leads to $g_{2}^{\prime}=c g_{1}^{\prime}, c \in \mathbb{R}-\{0\}$. Substituting this into (4.1) gives $\left(1+c^{2}\right) g_{1}^{\prime} g_{1}^{\prime \prime}=0$ or $g_{2}^{\prime \prime}=0$. This means that $K_{0}$ must be zero. Therefore $\beta$ becomes an isotropic line and the surface is a generalized cylinder with isotropic rulings.

Theorem 4.2. If the surface in $\mathbb{G}_{3}$ given by (4.3) has constant mean curvature $H_{0}$, then either it is a generalized cylinder with isotropic rulings $\left(H_{0}=0\right)$; or the generating curve $\beta$ is a Euclidean circle with radius $\frac{1}{2\left|H_{0}\right|}\left(H_{0} \neq 0\right)$.

Proof. Assume that the surface given by (4.3) has constant mean curvature $H_{0}$. Then we have the relation

$$
2 H_{0}=\frac{g_{2}^{\prime \prime}}{g_{1}^{\prime}} .
$$

This immediately implies that $H_{0}$ vanishes provided $\beta$ is an isotropic line. If $H_{0} \neq 0$, then we have from (4.7)

$$
g_{2}^{\prime \prime}=2 H_{0} g_{1}^{\prime} \text {. }
$$

Considering (4.8) into (4.1) gives

$$
g_{1}^{\prime \prime}=-2 H_{0} g_{2}^{\prime} .
$$

We may formulate the equations (4.8) and (4.9) as follows:

$$
\left\{\begin{array}{l}
g_{1}^{\prime \prime \prime}+4 H_{0}^{2} g_{1}^{\prime}=0 \\
g_{2}^{\prime \prime \prime}+4 H_{0}^{2} g_{2}^{\prime}=0
\end{array}\right.
$$

After solving (4.10) we obtain, up to suitable constants,

$$
\left\{\begin{array}{c}
g_{1}=\frac{c_{1}}{2\left|H_{0}\right|} \sin \left(2\left|H_{0}\right| u\right)+\frac{c_{2}}{2\left|H_{0}\right|} \cos \left(2\left|H_{0}\right| u\right) \\
g_{2}=\frac{c_{3}}{2\left|H_{0}\right|} \sin \left(2\left|H_{0}\right| v\right)+\frac{c_{4}}{2\left|H_{0}\right|} \cos \left(2\left|H_{0}\right| v\right)
\end{array}\right.
$$

where $c_{1}, \ldots, c_{4} \in \mathbb{R}$. Because $\left(g_{1}^{\prime}\right)^{2}+\left(g_{2}^{\prime}\right)^{2}=1$ we have $\left(c_{1}\right)^{2}+\left(c_{3}\right)^{2}=1,\left(c_{2}\right)^{2}+\left(c_{4}\right)^{2}=1$ and $c_{1} c_{2}+c_{3} c_{4}=0$. This means that $\beta$ is a Euclidean circle with radius $\frac{1}{2\left|H_{0}\right|}$.

Remark 4.1. It is easy to see from Theorem 4.2 that for a surface in $\mathbb{G}_{3}$ given by (4.3) $H=0$ implies $K=0$. 


\section{Constant Curvature Surfaces of Type 4}

In last section, we are interested in the surfaces generated by translating a space curve $\alpha=\alpha(u)=$ $\left(u, f_{1}(u), f_{2}(u)\right)$ and a planar non-isotropic curve $\beta=\beta(v)=(v, g(v), a v), a \in \mathbb{R}$. Since the torsion of $\alpha$ is different from zero, we have

$$
f_{1}^{\prime \prime} f_{2}^{\prime \prime \prime}-f_{1}^{\prime \prime \prime} f_{2}^{\prime \prime} \neq 0
$$

where $\frac{d f_{i}}{d u}=f_{i}^{\prime}$ and so on, $i=1,2$. Therefore the obtained translation surface is parameterized by

$$
r(u, v)=\left(u+v, f_{1}(u)+g(v), f_{2}(u)+a v\right) .
$$

By a calculation, the Gaussian curvature turns to

$$
K=\frac{g^{\prime \prime}\left[f_{1}^{\prime \prime}\left(f_{2}^{\prime}-a\right)^{2}-f_{2}^{\prime \prime}\left(f_{2}^{\prime}-a\right)\left(f_{1}^{\prime}-g^{\prime}\right)\right]}{\left[\left(f_{2}^{\prime}-a\right)^{2}+\left(f_{1}^{\prime}-g^{\prime}\right)^{2}\right]^{2}} .
$$

Theorem 5.1. If the surface in $\mathbb{G}_{3}$ given by (5.2) has constant Gaussian curvature $K_{0}$, then it is a generalized cylinder with non-isotropic rulings $\left(K_{0}=0\right)$.

Proof. Assume that $K_{0}=0$ and $g^{\prime \prime} \neq 0$. Then (5.3) turns to

$$
f_{1}^{\prime \prime}\left(f_{2}^{\prime}-a\right)-f_{2}^{\prime \prime}\left(f_{1}^{\prime}-g^{\prime}\right)=0 .
$$

Taking partial derivative of (5.4) with respect to $v$, we get $f_{2}^{\prime \prime}=0$ which is not possible due to (5.1). Hence, it follows $g^{\prime \prime}=0$, namely the surface is generalized cylinder with non-isotropic rulings. Next, we assume that $K_{0} \neq 0$. This immediately implies $g^{\prime \prime} \neq 0$. Dividing (5.3) with $g^{\prime \prime}$ and then taking its partial derivative with respect to $u$ leads to

$$
\left[\frac{d(A)}{d u}-\frac{d(B)}{d u}\left(f_{1}^{\prime}-g^{\prime}\right)-B f_{1}^{\prime \prime}\right]\left[C+\left(f_{1}^{\prime}-g^{\prime}\right)^{2}\right]-4\left[A-B\left(f_{1}^{\prime}-g^{\prime}\right)\right]\left[B+\left(f_{1}^{\prime}-g^{\prime}\right) f_{1}^{\prime \prime}\right]=0,
$$

where

$$
A=f_{1}^{\prime \prime}\left(f_{2}^{\prime}-a\right)^{2}, \quad B=f_{2}^{\prime \prime}\left(f_{2}^{\prime}-a\right), \quad C=\left(f_{2}^{\prime}-a\right)^{2} .
$$

(5.5) is a polynomial equation on $g^{\prime}$ and the leading coefficient which comes from the term $\left(g^{\prime}\right)^{3}$ is $\frac{d(B)}{d u}$. This must be zero, i.e. $B=B_{0} \in \mathbb{R}-\{0\}$. Thus (5.5) reduces to

$$
\left[\frac{d(A)}{d u}-B_{0} f_{1}^{\prime \prime}\right]\left[C+\left(f_{1}^{\prime}-g^{\prime}\right)^{2}\right]-4\left[A-B_{0}\left(f_{1}^{\prime}-g^{\prime}\right)\right]\left[B_{0}+\left(f_{1}^{\prime}-g^{\prime}\right) f_{1}^{\prime \prime}\right]=0 .
$$

Similarly, (5.7) is a polynomial equation on $g^{\prime}$ and the leading coefficient which comes from the term $\left(g^{\prime}\right)^{2}$ is

$$
\frac{d(A)}{d u}+3 B_{0} f_{1}^{\prime \prime}=0
$$

and the coefficient of the term $g^{\prime}$

$$
\left(\frac{d(A)}{d u}-B_{0} f_{1}^{\prime \prime}\right) f_{1}^{\prime}+2\left(-A f_{1}^{\prime \prime}+2 B_{0} f_{1}^{\prime} f_{1}^{\prime \prime}+B_{0}^{2}\right)=0 .
$$

Putting (5.8) into (5.9) gives

$$
\left(f_{1}^{\prime \prime}\right)^{2}-\left(f_{2}^{\prime \prime}\right)^{2}=0,
$$

which is a contradiction due to (5.1). Therefore the proof is completed.

By a calculation, the mean curvature turns to

$$
H=\frac{\left(f_{2}^{\prime}-a\right)\left(f_{1}^{\prime \prime}+g^{\prime \prime}\right)-\left(f_{1}^{\prime}-g^{\prime}\right) f_{2}^{\prime \prime}}{2\left[\left(f_{2}^{\prime}-a\right)^{2}+\left(f_{1}^{\prime}-g^{\prime}\right)^{2}\right]^{\frac{3}{2}}} .
$$


Theorem 5.2. There does not exist a minimal translation surface in $\mathbb{G}_{3}$ given by (5.2).

Proof. The proof is by contradiction. Then (5.10) reduces to

$$
\left(f_{2}^{\prime}-a\right)\left(f_{1}^{\prime \prime}+g^{\prime \prime}\right)-\left(f_{1}^{\prime}-g^{\prime}\right) f_{2}^{\prime \prime}=0 .
$$

The partial derivative of (5.11) with respect to $v$ yields

$$
f_{2}^{\prime \prime} g^{\prime \prime}+\left(f_{2}^{\prime}-a\right) g^{\prime \prime \prime}=0 .
$$

Because (5.1), $g^{\prime \prime}$ and $g^{\prime \prime \prime}$ in (5.12) cannot be linearly independent. Therefore it follows $g^{\prime \prime \prime}=c_{1} g^{\prime \prime}, c_{1} \in \mathbb{R}$ and an integration yields

$$
g^{\prime \prime}=c_{1} g^{\prime}+c_{2}, c_{2} \in \mathbb{R} .
$$

Substituting (5.13) into (5.11) gives a polynomail equation on $g^{\prime}$ of degree one, i.e.

$$
\left(f_{2}^{\prime}-a\right)\left(f_{1}^{\prime \prime}+c_{1} g^{\prime}+c_{2}\right)-\left(f_{1}^{\prime}-g^{\prime}\right) f_{2}^{\prime \prime}=0 .
$$

The fact that the coefficients must be zero gives

$$
c_{1}\left(f_{2}^{\prime}-a\right)+f_{2}^{\prime \prime}=0
$$

and

$$
\left(f_{2}^{\prime}-a\right)\left(f_{1}^{\prime \prime}+c_{2}\right)-f_{1}^{\prime} f_{2}^{\prime \prime}=0 .
$$

Note that $c_{1} \neq 0$ due to $f_{2}^{\prime \prime} \neq 0$ in (5.14). Putting (5.14) into (5.15) leads to

$$
f_{1}^{\prime \prime}+c_{1} f_{1}^{\prime}+c_{2}=0 .
$$

From (5.14) and (5.16) we easily get $f_{2}^{\prime \prime \prime}=-c_{1} f_{2}^{\prime \prime}$ and $f_{1}^{\prime \prime \prime}=-c_{1} f_{1}^{\prime \prime}$. This contradicts with (5.1).

Theorem 5.3. If the surface in $\mathbb{G}_{3}$ given by (5.2) has nonzero constant mean curvature $H_{0}$, then it is a generalized cylinder with non-isotropic rulings whose the base curve satisfies the following equation, up to a suitable constant and a translation of $u$

$$
f_{1}(u)=g_{0} u-\frac{1}{2 H_{0}} \sqrt{1-4 H_{0}\left(f_{2}(u)-a u\right)^{2}},
$$

where $g^{\prime}=g_{0} \in \mathbb{R}$.

Proof. The partial derivative of (5.10) with respect to $v$ gives

$$
-6 H_{0}\left[\left(f_{2}^{\prime}-a\right)^{2}+\left(f_{1}^{\prime}-g^{\prime}\right)^{2}\right]^{\frac{1}{2}}\left(f_{1}^{\prime}-g^{\prime}\right) g^{\prime \prime}=\left(f_{2}^{\prime}-a\right) g^{\prime \prime \prime}+f_{2}^{\prime \prime} g^{\prime \prime} .
$$

To solve (5.17), we have two cases:

1. $g^{\prime}=g_{0}, g_{0} \in \mathbb{R}$. (5.10) turns to

$$
2 H_{0}=\frac{\left(f_{2}^{\prime}-a\right) f_{1}^{\prime \prime}-\left(f_{1}^{\prime}-g_{0}\right) f_{2}^{\prime \prime}}{\left[\left(f_{2}^{\prime}-a\right)^{2}+\left(f_{1}^{\prime}-g_{0}\right)^{2}\right]^{\frac{3}{2}}}
$$

(5.18) can be rewritten as

$$
2 H_{0}\left(f_{2}^{\prime}-a\right)=\frac{\left(\frac{f_{1}^{\prime}-g_{0}}{f_{2}^{\prime}-a}\right)^{\prime}}{\left[1+\left(\frac{f_{1}^{\prime}-g_{0}}{f_{2}^{\prime}-a}\right)^{2}\right]^{\frac{3}{2}}}
$$

An integration of (5.19) with respect to $u$ gives

$$
2 H_{0}\left(f_{2}-a u+c\right)=\frac{\frac{f_{1}^{\prime}-g_{0}}{f_{2}^{\prime}-a}}{\left(1+\left(\frac{f_{1}^{\prime}-g_{0}}{f_{2}^{\prime}-a}\right)^{2}\right)^{\frac{1}{2}}}, c \in \mathbb{R} .
$$


Up to a translation on $u$, we may assume $c=0$. (5.20) can be rewritten as

$$
\frac{4 H_{0}\left(f_{2}-a u\right)^{2}}{1-4 H_{0}\left(f_{2}-a u\right)^{2}}=\left(\frac{f_{1}^{\prime}-g_{0}}{f_{2}^{\prime}-a}\right)^{2}
$$

or

$$
f_{1}^{\prime}-g_{0}=\frac{2 H_{0}\left(f_{2}-a u\right)\left(f_{2}^{\prime}-a\right)}{\sqrt{1-4 H_{0}\left(f_{2}-a u\right)^{2}}} .
$$

Up to suitable constant, an integration of (5.21) with respect to $u$ gives

$$
f_{1}=g_{0} u-\frac{1}{2 H_{0}} \sqrt{1-4 H_{0}\left(f_{2}-a u\right)^{2}} .
$$

2. $g^{\prime \prime} \neq 0$. (5.17) can be rewritten by dividing $g^{\prime \prime}$ as

$$
6 H_{0}\left[\left(f_{2}^{\prime}-a\right)^{2}+\left(f_{1}^{\prime}-g^{\prime}\right)^{2}\right]^{\frac{1}{2}}\left(f_{1}^{\prime}-g^{\prime}\right)=\left(f_{2}^{\prime}-a\right) \frac{g^{\prime \prime \prime}}{g^{\prime \prime}}+f_{2}^{\prime \prime}
$$

The partial derivative of (5.22) with respect to $v$ gives

$$
\frac{\left(f_{2}^{\prime}-a\right)^{2}+2\left(f_{1}^{\prime}-g^{\prime}\right)^{2}}{\left(f_{2}^{\prime}-a\right)\left[\left(f_{2}^{\prime}-a\right)^{2}+\left(f_{1}^{\prime}-g^{\prime}\right)^{2}\right]^{\frac{1}{2}}}=\frac{1}{6 H_{0} g^{\prime \prime}}\left(\frac{g^{\prime \prime \prime}}{g^{\prime \prime}}\right)^{\prime} \text {. }
$$

By again taking partial derivative of (5.23) with respect to $u$ we derive a polynomial equation on $g^{\prime}$. The leading coefficient which comes of the term $\left(g^{\prime}\right)^{4}$ is $-4\left(f_{2}^{\prime}-a\right) f_{2}^{\prime \prime}$. This can not be vanish due to (5.1) and therefore we achieve a contradiction.

Remark 5.1. It is easy to see from Theorem 5.3 that for a surface in $\mathbb{G}_{3}$ given by (5.2) $H=$ const. $\neq 0$ implies $K=0$.

\section{Conclusions}

This study was devoted to obtain translation surfaces in $\mathbb{G}_{3}$ with $K=$ const. and $H=$ const. when at least one of the generating curves is planar. In this sense it is still an open problem to find the translation surfaces in $\mathbb{G}_{3}$ with $K=$ const. and $H=$ const. when both of generating curves are non-planar.

\section{References}

[1] Abdel-Baky, R.A. and Unluturk, Y., A study on classification of translation surfaces in pseudo-Galilean 3-space. J. Coupl. Syst. Multi. Dynm. 6 (2018), no. 3, 233-240.

[2] Aydin, M.E., Mihai, A., Ogrenmis, A.O. and Ergut, M., Geometry of the solutions of localized induction equation in the pseudo-Galilean space. Adv. Math. Phys. vol. 2015, Article ID 905978, 7 pages, 2015. doi:10.1155/2015/905978.

[3] Aydin, M.E., Ogrenmis, A.O. and Ergut, M., Classification of factorable surfaces in the pseudo-Galilean space. Glas. Mat. Ser. III, 50(70), 441-451, 2015.

[4] Cakmak, A., Karacan, M.K., Kiziltug, S. and Yoon D. W., Translation surfaces in the 3-dimensional Galilean space satisfying $\Delta^{I I} x_{i}=x_{i}$, Bull. Korean Math. Soc. 54 (2017), no. 4, 1241-1254.

[5] Darboux, J. G., Theorie Generale des Surfaces. Livre I, Gauthier-Villars, Paris, 1914.

[6] Dede, M., Tubular surfaces in Galilean space. Math. Commun. 18 (2013), 209-217.

[7] Dede, M., Tube surfaces in pseudo-Galilean space. Int. J. Geom. Methods Mod. Phys. 13 (2016), no. 5, 1650056, 10 pp.

[8] Dede, M., Ekici, C., Goemans, W. and Unluturk, Y., Twisted surfaces with vanishing curvature in Galilean 3-space. Int. J. Geom. Methods Mod. Phys. 15(1) (2018), 1850001, 13pp.

[9] Dede, M., Ekici, C. and Goemans, W., Surfaces of revolution with vanishing curvature in Galilean 3-space. J. Math. Phys. Anal. Geom. 14 (2018), no. 2, 141-152.

[10] Dillen, F., Goemans, W. and Woestyne, Van De I., Translation surfaces of Weigarten type in 3-space. Bull. Transilv. Univ. Brasov Ser. III, Math. Inform. Phys. 1 (2008), no. 50, 109-122. 
[11] Dillen, F., Verstraelen, L. and G. Zafindratafa, A generalization of the translation surfaces of Scherk. Differential Geometry in Honor of Radu Rosca: Meeting on Pure and Applied Differential Geometry, Leuven, Belgium, 1989, KU Leuven, Departement Wiskunde (1991), pp. 107-109.

[12] Divjak, B. and Milin-Sipus, Z., Special curves on ruled surfaces in Galilean and pseudo-Galilean spaces. Acta Math. Hungar. 98 (2003), 175-187.

[13] Erjavec, Z., Divjak, B. and Horvat, D., The general solutions of Frenet's system in the equiform geometry of the Galilean, pseudo-Galilean, simple isotropic and double isotropic space. Int. Math. Forum. 6 (2011), no. 1, 837-856.

[14] Erjavec, Z., On generalization of helices in the Galilean and the pseudo-Galilean space. J. Math. Research 6 (2014), no. 3, 39-50.

[15] Giering O., Vorlesungen uber hohere Geometrie, Friedr. Vieweg \& Sohn, Braunschweig, Germany, 1982.

[16] Gray A., Modern Differential Geometry of Curves and Surfaces with Mathematica. CRC Press LLC, 1998.

[17] Inoguchi, J., Lopez, R. and Munteanu M.I., Minimal translation surfaces in the Heisenberg group Nil 3 . Geom. Dedicata 161 (2012), 221-231.

[18] Kazan, A. and Karadag, H.B., Twisted Surfaces in the Pseudo-Galilean Space. NTMSCI 5 (2017), no.4, 72-79.

[19] Liu, H., Translation surfaces with constant mean curvature in 3-dimensional spaces. J. Geom. 64 (1999), no. 1-2, 141-149.

[20] Liu, H. and Yu, Y., Affine translation surfaces in Euclidean 3-space. In: Proceedings of the Japan Academy, Ser. A, Mathematical Sciences, vol. 89, pp. 111-113, Ser. A (2013).

[21] Liu, H. and Jung, S.D., Affine translation surfaces with constant mean curvature in Euclidean 3-space. J. Geom. 108 (2017), no. 2, 423-428.

[22] Lopez, R. and Munteanu M.I., Minimal translation surfaces in Sol 3. J. Math. Soc. Japan 64 (2012), no. 3, $985-1003$.

[23] Lopez, R., Moruz, M., Translation and homothetical surfaces in Euclidean space with constant curvature. J. Korean Math. Soc. 52 (2015), no. 3, 523-535.

[24] Lopez, R., Minimal translation surfaces in hyperbolic space. Beitr. Algebra Geom. 52 (2011), no. 1, $105-112$.

[25] Milin-Sipus, Z., Ruled Weingarten surfaces in the Galilean space.Period. Math. Hungar. 56 (2008), $213-225$.

[26] Milin-Sipus, Z. and Divjak, B., Some special surfaces in the pseudo-Galilean Space. Acta Math. Hungar. 118 (2008), $209-226$.

[27] Milin-Sipus, Z. and Divjak, B., Translation surface in the Galilean space. Glas. Mat. Ser. III 46 (2011), no. 2, $455-469$.

[28] Milin-Sipus, Z., and Divjak, B., Surfaces of constant curvature in the pseudo-Galilean space. Int. J. Math. Sci., 2012, Art ID375264, 28pp.

[29] Milin-Sipus, Z., On a certain class of translation surfaces in a pseudo-Galilean space. Int. Mat. Forum 6 (2012), no. 23, $1113-1125$.

[30] Milin-Sipus, Z., Translation surfaces of constant curvatures in a simply isotropic space. Period. Math. Hung. 68 (2014), $160-175$.

[31] Moruz, M. and Munteanu M.I., Minimal translation hypersurfaces in $\mathbb{E}^{4}$. J. Math. Anal. Appl. 439 (2016), no. 2, 798-812.

[32] Munteanu M.I., Palmas, O. and Ruiz-Hernandez, G., Minimal translation hypersurfaces in Euclidean spaces. Mediterranean J. Math. 13 (2016), 2659-2676.

[33] Onishchick, A. and Sulanke, R., Projective and Cayley-Klein Geometries. Springer, 2006.

[34] Pavkovic, B.J. and Kamenarovic, I., The equiform differential geometry of curves in the Galilean space $\mathbb{G}_{3}$. Glasnik Math. 22 (1987), no. 42, 449-457.

[35] Roschel, O., Die Geometrie des Galileischen Raumes. Forschungszentrum Graz, Mathematisch-Statistische Sektion, Graz, 1985.

[36] Roschel, O., Torusflachen des Galileischen Raumes. Studia Sci. Math. Hungarica, 23 (1988), no. 3-4, 401-410.

[37] Scherk, H.F., Bemerkungen uber die kleinste Flache innerhalb gegebener Grenzen. J. Reine Angew. Math. 13 (1835), 185-208.

[38] Seo, K., Translation Hypersurfaces with constant curvature in space forms. Osaka J. Math. 50 (2013), 631-641.

[39] Sun, H., On affine translation surfaces of constant mean curvature. Kumamoto J. Math. 13 (2000), $49-57$.

[40] Verstraelen, L., Walrave, J. and Yaprak, S., The minimal translation surfaces in Euclidean space. Soochow J. Math. 20 (1994), 77-82.

[41] Yang, D. and Fu, Y., On affine translation surfaces in affine space. J. Math. Anal. Appl. 440 (2016), no. 2, 437-450.

[42] Yoon, D.W., Minimal Translation Surfaces in $\mathbb{H}^{2} \times \mathbb{R}$. Taiwanese J. Math. 17(5) (2013), 1545-1556.

[43] Yoon, D.W., Classification of rotational surfaces in pseudo-Galilean space. Glas. Mat. Ser. III 50 (2015), no. 2, $453-465$.

\section{Affiliations}

MUHITTIN EVREN AYDIN

ADDRESS: Firat University, Dept. of Mathematics, 23200, Elazig-Turkey

E-MAIL: meaydin@firat.edu.tr

ORCID ID: orcid.org/0000-0001-9337-8165

Mihriban AlyamaÇ KulahÇI

ADDRESS: Firat University, Dept. of Mathematics, 23200, Elazig-Turkey

E-MAIL: mihribankulahci@gmail.com

ORCID ID: orcid.org/0000-0002-8621-5779

\section{AlPER OSMAN ÖĞRENMIŞ}

ADDRESS: Firat University, Dept. of Mathematics, 23200, Elazig-Turkey

E-MAIL: aogrenmis@firat.edu.tr

ORCID ID: orcid.org/0000-0001-5008-2655 